\title{
Internações por Condições Sensíveis à Atenção Primária no Estado de São Paulo
}

\author{
Internment for Conditions Suitable \\ for Primary Healthcare in Sao Paulo State
}

Tania Cristina Morais Santa Barbara Rehem ${ }^{1}$

Emiko Yoshikawa Egry ${ }^{2}$

${ }^{1}$ Escola de Enfermagem, Universidade de São Paulo (USP). Av. Dr. Enéas de Carvalho Aguiar 419. 05403-000 São Paulo SP. tania.rehem@usp.br ${ }^{2}$ Departamento de Enfermagem em Saúde Coletiva, Escola de Enfermagem, Universidade de São Paulo (USP)
Abstract Internments suitable for Primary Healthcare constitute a new indicator used in some countries to indirectly measure the resolution capability of Primary Healthcare. The scope of this study is to present the panorama of the internments suitable for primary healthcare in Sao Paulo State. An ecological descriptive study was carried out for the period from 2000 to 2007. The data were generated by patient's county of residence and grouped by Regional Health Departments. The Information Systems of the Health Ministry and the population estimates of the Brazilian Institute of Geography and Statistics (IBGE) were used to obtain the data of all patients. The internments suitable for primary healthcare in the state follow a tendency toward reduction suggesting a connection with the adoption of the Family Health Program. A reduction in internments for diabetes was detected and attention was drawn to the increase of internment for illnesses related to prenatal care and childbirth. In light of the recent publication of the Brazilian Diagnostics List, studies that analyze the statistics from it can be very useful for managers involved with primary care, and can serve as benchmarks for quality and contribute to assessment for the implementation of health policies in Brazil.

Key words Health policies, Primary healthcare
Resumo Internações Sensíveis à Atenção Primária se constitui num novo indicador, utilizado em alguns países, para medir indiretamente o funcionamento e a capacidade de resolução da Atenção Primária. Este estudo tem como objetivo apresentar o panorama das internações sensiveis à atenção primária no Estado de São Paulo. Trata-se de um estudo descritivo ecológico, realizado no período de 2000 a 2007. Os dados foram gerados por município de residência do paciente e agregados por Departamentos Regionais de Saúde. Para obtenção dos mesmos foram utilizados os Sistemas de Informação do Ministério da Saúde e estimativa populacional do IBGE. As internações sensiveis à atenção primária no Estado seguem uma tendência de redução sugerindo relação com a adoção da Estratégia de Saúde da Família. Observa-se redução nas internações por diabetes e chama atenção o aumento de internações por doenças relacionadas ao pré-natal e parto. Considerando a publicação recente da Lista Brasileira de Diagnósticos, os estudos que se utilizem dela podem ser de grande valia para os gestores envolvidos com a atenção primária, podendo funcionar como balizadores de sua qualidade contribuindo para avaliação da implantação das políticas de saúde no Brasil. Palavras-chaves Políticas de saúde, Atenção primária 


\section{Introdução}

Na década de 80, Billings e Teicholz ${ }^{1}$ desenvolveram o conceito de hospitalizações potencialmente evitáveis ou condições sensíveis à atenção ambulatorial, como um reflexo indireto de problemas com o acesso e a efetividade dos cuidados primários.

A partir de então estudos vêm sendo desenvolvidos utilizando dados de internações potencialmente evitáveis mostrando sua estreita relação com as características dos sistemas de saúde, especialmente com a política de atenção primária ${ }^{2-6}$.

Para Caminal e Casanova ${ }^{7}$ a hospitalização por problemas de saúde susceptíveis de cuidados pela atenção primária, denominado Ambulatory Care Sensitive Conditions (ACSC), constitui um novo indicador de atividade hospitalar, desenvolvido na última década, que pretende servir de medida da efetividade da atenção primária à saúde para a atenção de determinados problemas de saúde. Para as autoras, o fundamento que sustenta este indicador é que uma elevada capacidade de resolução por parte da atenção primária deveria implicar uma diminuição dos ingressos hospitalares, pelo incremento das medidas preventivas, assim como da melhoria dos tratamentos ambulatoriais.

No III Seminário Internacional Sobre Atenção Primária - Saúde da Família, realizado no Brasil em 2007, esse indicador foi apresentado como Internações Sensíveis à Atenção Básica (ISAB), sendo definida por Macinko ${ }^{8}$ como aquelas que deveriam ser evitadas caso os serviços de atenção básica fossem efetivos e acessíveis. Estas podem envolver, entre outras, complicações não controladas de uma doença crônica ou outras condições; e condições relacionadas com a falta de acesso aos serviços preventivos ou às rotinas.

Ainda segundo esse autor, dentre outras questões, deve-se estudar as ISAB para identificar problemas de acesso aos serviços de atenção básica e de qualidade dos serviços - como exemplo deste último o controle inadequado de doenças crônicas; para avaliar políticas/ reformas específicas do sistema de saúde, por exemplo, expansão da Saúde da Família; e usar como um indicador "genérico" para monitorar o desempenho (efetividade) do sistema de atenção básica.

De acordo com a revisão de literatura, o indicador ACSC teve origem nos Estados Unidos ${ }^{2}$ e a partir daí foram realizadas adaptações de forma a contemplar especificidades locais, dependendo do contexto a ser analisado. Isto pode ser observado no estudo realizado onde foi aplicada a lista americana para validação no contexto europeu’.
Macinko ${ }^{8}$ cita como limitações para o uso deste novo indicador, as características exógenas à Atenção Primária, tais como o fato de poder levar anos para se detectar problemas em áreas pequenas, mudanças pontuais e estabelecimentos de tendências, além do fato das ISAP dependerem das informações hospitalares, ou seja, mortes ou outras complicações que não aconteçam nos hospitais não são refletidas. Pode-se acrescentar também a limitação de se usar dados secundários e a da confiabilidade nos diagnósticos.

$\mathrm{Na}$ Espanha, entre as estratégias propostas para melhorar a utilidade desse indicador está a busca de uma maior especificidade e análise do mesmo por município².

Os profissionais da atenção primária à saúde (APS) têm resistido a que sua prática clínica seja avaliada por meio de um indicador de atividade hospitalar ${ }^{7}$. Esta resistência, segundo a autora, baseia-se na existência de fatores determinantes da hospitalização que fogem do controle dos profissionais da APS. Assim, este indicador teria limitações para ser utilizado na avaliação do primeiro nível de assistência. Relata ainda que os fatores mais relevantes que podem condicionar a hospitalização são as características dos pacientes, a variabilidade da prática clínica hospitalar e as políticas de admissão dos centros.

Por outro lado, Starfield ${ }^{10}$ relata que internações por condições sensíveis à atenção primária é um dos indicadores genéricos para avaliação de desfechos de atenção primária em âmbito populacional. Nesse sentido, as taxas de internações por condições sensíveis à atenção primária têm sido utilizadas em alguns países como um indicador indireto de avaliação do acesso e da qualidade da atenção.

No Brasil, a primeira lista nacional das internações por condições sensíveis à atenção primária (ISAP), foi elaborada no ano de 2007, com base nas listas já existentes em algumas Secretarias de Estado da Saúde, revisão bibliográfica de trabalhos internacionais, e reunião de consenso entre pesquisadores, gestores e especialistas no tema. Em seguida esta lista foi encaminhada para a Sociedade Brasileira de Saúde da Família e comunidade e para consulta publica ${ }^{11}$.

Estava contemplado nesta lista um conjunto de diagnósticos para o qual a atenção primária efetiva reduziria o número de internações. Isto é, as atividades próprias da atenção primária, tais como a prevenção de doenças; o diagnóstico precoce e o tratamento oportuno de patologias agudas; e o controle e acompanhamento de patologias crônicas ${ }^{11}$. 
Doenças (CID-10).

A partir daí, a Secretaria de Atenção à Saúde do Ministério da Saúde (SAS/MS) considerando, entre outros aspectos, o impacto da atenção primária em saúde na redução das internações por condições sensíveis a este nível de atenção em vários países, e a possibilidade de incluir indicadores da atividade hospitalar para serem utilizados como medida indireta do funcionamento da atenção básica brasileira e da Estratégia Saúde da Família publicou a versão final da lista na forma de anexo da Portaria de n. 221, de 17 de abril de $2008^{13}$.

O MS estabelece ainda que a lista deverá ser utilizada como instrumento de avaliação da atenção primária e ou da utilização da atenção hospitalar, podendo ser aplicada para avaliar o desempenho do sistema de saúde nos âmbitos $\mathrm{Na}$ cional, Estadual e Municipal.

A atenção primária, segundo Starfield ${ }^{14}$, em sua forma mais desenvolvida é o primeiro contato com o sistema de saúde e o local responsável pela organização do cuidado à saúde dos indivíduos, suas famílias e da população ao longo do tempo. Evidências demonstram que esse nível de atenção tem capacidade para responder a $85 \%$ das necessidades em saúde, por meio da realização de serviços preventivos, curativos, reabilitadores e de promoção da saúde; integrando os cuidados quando existe mais de um problema; lidando com o contexto de vida e influenciando as respostas das pessoas aos seus problemas de saúde.

Embora o quadro evolutivo apresentado pelo crescimento da oferta de serviços da atenção primária indique uma ampliação de acesso a esses serviços, permanecem alguns desafios no campo do atendimento das necessidades de saúde da população que se renovam a cada etapa de implantação do SUS. Dentre estes desafios pode ser citada a fragmentação das políticas e programas de saúde, para a qual, o pacto pela saúde traz como uma das propostas de superação a estruturação de uma rede solidária e regionalizada de ações e serviços que qualifiquem o processo de gestão.

A estruturação dessas redes deve ter como ponto de partida a organização da atenção pri- mária, situada no centro desta rede, como ordenadora de fluxos e contra fluxos de pessoas para os demais níveis do sistema, à exceção dos casos de urgência e emergência.

No que diz respeito a estes fluxos, em muitas situações onde a atenção primária não é resolutiva, a demanda por internações hospitalares provavelmente inclui uma proporção de casos com diagnósticos sensíveis à atenção primária, $\mathrm{e}$ que assim poderiam ser resolvidas neste nível de atenção.

Nesse contexto, considerando a Portaria SAS/ MS de n. 221, recém-publicada, este artigo tem como objetivo, apresentar o panorama das internações por condições sensíveis à atenção primária registradas no Estado de São Paulo, no período de 2000 a 2007, segundo os Departamentos Regionais de Saúde (DRS) do Estado.

\section{Metodologia}

Foi realizado um estudo descritivo ecológico considerando como unidade de análise uma população pertencente a uma área geográfica definida.

Segundo Medronho et al. ${ }^{15}$, os estudos ecológicos são frequentemente realizados combinando-se bases de dados referentes a grandes populações. Em função disto são geralmente estudos mais baratos e mais rápidos que aqueles envolvendo o indivíduo como unidade de análise ou um grupo de pessoas.

Foram levantados os dados de internação hospitalar de pacientes residentes, registrados no Sistema de Informações Hospitalares (SIH) do SUS, no período de 2000 a 2007, segundo a Diretoria Regional de Saúde do Estado de São Paulo, bem como a cobertura da população por Equipes de Saúde da Família, a partir dos dados do Sistema de Informações da Atenção Básica (SIAB).

O SIH-SUS é o sistema que processa as autorizações de internações hospitalares (AIHs), disponibilizando informações sobre recursos destinados a cada hospital que integra a rede do SUS, as principais causas de internações no Brasil, a relação dos procedimentos mais frequentes realizados mensalmente em cada hospital, município e estado, a quantidade de leitos existentes para cada especialidade e o tempo médio de permanência do paciente no hospital. Suas informações facilitam as atividades de Controle e Avaliação e estão disponibilizadas via Internet por meio de produtos desenvolvidos pelo DATASUS.

O SIAB é o principal instrumento de monitoramento das ações da Saúde da Família, sendo 
sua base de dados disponibilizada na internet como parte das ações estratégicas da política definida pelo Ministério da Saúde que tem como objetivo fornecer informações que subsidiem a tomada de decisão pelos gestores do SUS, e a instrumentalização pelas instâncias de Controle Social, publicando, assim, os dados para o uso de todos os atores envolvidos na consolidação do SUS.

A seleção das Internações por Condições Sensíveis à Atenção Primária foi feita com base na Lista Brasileira publicada pelo Ministério da Saúde, por meio da Portaria SAS/MS no 221, de 17 de abril de 2008. Essa Lista é composta por 19 grupos de causas, com 74 diagnósticos classificados de acordo com a décima Revisão da Classificação Internacional de Doenças - CID10 (Quadro 1).

A partir da seleção no Sistema de Informações Hospitalares do SUS - SIH/SUS dos códigos acima referidos, foi gerado um arquivo de definição (DEF) para tabulação das causas sensíveis, utilizando o aplicativo Tabwin - Versão 3.5 - desenvolvido pelo DATASUS/MS. Para análise das internações sensíveis à atenção primária foram utilizados dois indicadores: um relativo à participação das internações sensíveis à atenção primária no total de internações e o outro relativo à proporção de internações por condições sensíveis à atenção primária por habitantes.

Para o cálculo do indicador relativo à participação das internações sensíveis à atenção primária, utilizou-se a fórmula: total de internações no SUS por essas causas, segundo DRS de residência, dividido pelo total de internações de pacientes residentes, no mesmo local e no período selecionado (2000 a 2007), multiplicado por 100. As internações sensíveis foram analisadas por grupos de causas, segundo municípios, agregando em seguida por DRS e estado.

Para o cálculo da proporção de internações por condições sensíveis à atenção primária por habitantes, utilizou-se a fórmula: total de inter-

Quadro 1. Lista de Condições Sensíveis à Atenção Primária

\begin{tabular}{|c|c|}
\hline Diagnóstico CID 10 & Códigos Selecionados \\
\hline $\begin{array}{l}\text { 1. Doenças preveníveis por imunização e condições } \\
\text { sensíveis }\end{array}$ & $\begin{array}{l}\text { A37; A36; A33 a A35; B26; B06; B05; A95; B16; } \\
\text { G00.0; A17.0 A19; A15.0 a A15.3; A16.0 a A16.2, } \\
\text { A15.4 a A15.9, A16.3 a A16.9, A17.1 a A17.9; } \\
\text { A18; I00 a I02; A51 a A53; B50 a B54 }\end{array}$ \\
\hline 2. Gastroenterites infecciosas e complicações & E86; A00 a A09 \\
\hline 3. Anemia & D50 \\
\hline 4. Deficiências nutricionais & E40 a E46; E50 a E64 \\
\hline 5. Infecções de ouvido, nariz e garganta & H66; J00; J01; J02; J03; J06; J31 \\
\hline 6. Pneumonias bacterianas & J13; J14; J15.3, J15.4; J15.8, J15.9; J18.1 \\
\hline 7. Asma & J45, J46 \\
\hline 8. Doenças pulmonares & $\mathrm{J} 20, \mathrm{~J} 21 ; \mathrm{J} 40 ; \mathrm{J} 41 ; \mathrm{J} 42 ; \mathrm{J} 43 ; \mathrm{J} 47 ; \mathrm{J} 44$ \\
\hline 9. Hipertensão & I10; I11 \\
\hline 10. Angina & $\mathrm{I} 20$ \\
\hline 11. Insuficiência cardíaca & I50; J81 \\
\hline 12. Doenças cerebrovasculares & I63 a I67; I69, G45 a G46 \\
\hline 13. Diabetes melitus & $\begin{array}{l}\text { E10.0, E10.1, E11.0, E11.1, E12.0, E12.1;E13.0, } \\
\text { E13.1; E14.0, E14.1; E10.2 a E10.8, E11.2 a } \\
\text { E11.8; E12.2 a E12.8;E13.2 a E13.8; E14.2 a } \\
\text { E14.8; E10.9, E11.9; E12.9, E13.9; E14.9 }\end{array}$ \\
\hline 14. Epilepsias & G40, G41 \\
\hline 15. Infecção no rim e trato urinário & N10; N11; N12; N30; N34; N39.0 \\
\hline 16. Infecção da pele e tecido subcutâneo & A46; L01; L02; L03; L04; L08 \\
\hline 17. Doença inflamatória órgãos pélvicos femininos & N70; N71; N72; N73; N75; N76 \\
\hline 18. Úlcera gastrointestinal & K25 а K28, K92.0, K92.1, K92.2 \\
\hline 19. Doenças relacionadas ao pré-natal e parto & O23; A50; P35. \\
\hline
\end{tabular}

Fonte: Portaria SAS/MS n. 221, de 17 de abril de 2008. 
nações ocorrida em pacientes residentes no município, dividido pela população total do município segundo estimativa populacional elaborada pelo IBGE para o Tribunal de Contas da União (TCU), multiplicado por 1000.

Para o cálculo da cobertura da população por Equipes de Saúde da Família, foi considerado o número de equipes de saúde da família e a média de 3.450 pessoas, com limitador de $100 \%$ de cobertura pelos municípios (DAB/SAS/MS). No denominador se utilizou a estimativa populacional elaborada pelo IBGE para o Tribunal de Contas da União (TCU).

Outra análise realizada foi a relação entre a cobertura da População por Equipes de Saúde da Família e Participação das Internações por Causas Sensíveis à Atenção Primária no Total das Internações de Pacientes Residentes, segundo DRS.

Foi realizada também a análise dos gastos realizados pelo SUS com internações por causas sensíveis à atenção primária, por município, agregado por DRS e estado, obtidos do SIH/SUS.

Os dados foram gerados por município de residência do paciente e agregados por Departamentos Regionais de Saúde, em um total de 17 DRS.

\section{Resultados}

Segundo dados do SIH/SUS o número de internações registradas no SUS/SP, comparando-se os dados de 2000 e 2007, sofreu um aumento de $4 \%$ com um acréscimo de 94.994 internações ano, às custas do crescimento ocorrido nos DRS de Araçatuba (22,97\%), Grande São Paulo (19,64\%), Sorocaba $(2,61 \%)$, Franca $(2,35 \%)$, Presidente Prudente (1,52\%) e Ribeirão Preto (0,30\%). Entre os DRS que apresentaram redução merecem destaque os DRS de Barretos (16,58\%), Marília (16,13\%) e São João da Boa Vista (12,18\%).

Entretanto a frequência das internações sensíveis à atenção primária neste mesmo período (Tabela 1) diminui no estado (de 435.594 em 2000 para 429.070 em 2007). Ao analisar por DRS verifica-se redução, exceto nos DRS da Grande São Paulo, Araçatuba e Ribeirão Preto. A maior redução ocorreu no DRS de Barretos (29,95\%), seguido de Araraquara e Taubaté.

Ao considerar o número de internações por Causas Sensíveis à Atenção Primária (Tabela 2), observa-se que a frequência de internação de pacientes residentes por CSAP por $1.000 \mathrm{hab} / \mathrm{ano}$, reduz mais ainda, permanecendo elevada apenas em dois DRS: Grande São Paulo e Araçatuba. A maior redução foi observada em Taubaté

Tabela 1. Número de Internações por Condições Sensíveis à Atenção Primária Registradas no SUS, segundo DRS de Residência do Paciente. São Paulo, 2000 a 2007.

\begin{tabular}{|c|c|c|c|c|c|c|c|c|c|}
\hline \multirow{2}{*}{ DRS } & \multicolumn{8}{|c|}{ Frequência de Internação Paciente Residente por CSAP } & \multirow{2}{*}{$\begin{array}{l}\text { Variação \% } \\
2007 / 2000\end{array}$} \\
\hline & 2000 & 2001 & 2002 & 2003 & 2004 & 2005 & 2006 & 2007 & \\
\hline Barretos & 9.859 & 9.799 & 9.647 & 9.203 & 8.453 & 8.981 & 8.587 & 6.906 & $-29,95$ \\
\hline Araraquara & 15.206 & 15.394 & 14.219 & 14.508 & 13.299 & 13.202 & 11.863 & 10.926 & $-28,15$ \\
\hline Taubaté & 28.351 & 28.271 & 28.690 & 27.908 & 25.325 & 23.800 & 21.353 & 20.593 & $-27,36$ \\
\hline Franca & 12.518 & 12.518 & 11.193 & 11.160 & 10.790 & 9.972 & 9.988 & 9.348 & $-25,32$ \\
\hline Campinas & 40.801 & 38.274 & 38.660 & 38.111 & 37.369 & 35.671 & 32.707 & 31.278 & $-23,34$ \\
\hline Piracicaba & 14.053 & 13.691 & 13.232 & 13.755 & 14.634 & 12.704 & 11.546 & 10.884 & $-22,55$ \\
\hline S João B Vista & 16.893 & 17.375 & 16.432 & 15.657 & 14.721 & 14.388 & 14.198 & 13.478 & $-20,22$ \\
\hline S José R Preto & 36.883 & 38.055 & 36.005 & 33.575 & 34.715 & 34.999 & 32.252 & 29.558 & $-19,86$ \\
\hline Marília & 26.527 & 26.119 & 22.542 & 23.296 & 22.269 & 22.069 & 22.103 & 21.899 & $-17,45$ \\
\hline Bauru & 29.413 & 31.350 & 27.769 & 27.909 & 27.486 & 27.060 & 26.699 & 24.978 & $-15,08$ \\
\hline Sorocaba & 27.846 & 28.850 & 28.847 & 29.088 & 29.174 & 27.784 & 26.662 & 25.114 & $-9,81$ \\
\hline B. Santista & 16.036 & 15.676 & 15.869 & 15.041 & 16.390 & 14.664 & 15.029 & 14.595 & $-8,99$ \\
\hline Registro & 2.709 & 2.876 & 2.485 & 2.631 & 2.659 & 2.464 & 2.544 & 2.582 & $-4,69$ \\
\hline P. Prudente & 13.527 & 13.192 & 14.405 & 14.041 & 13.637 & 13.634 & 15.087 & 13.514 & $-0,10$ \\
\hline Ribeirão Preto & 16.201 & 15.960 & 17.065 & 18.593 & 18.670 & 17.148 & 16.873 & 16.279 & 0,48 \\
\hline Araçatuba & 11.375 & 12.755 & 12.974 & 13.002 & 11.908 & 12.091 & 11.776 & 11.902 & 4,63 \\
\hline Grande S Paulo & 117.396 & 114.483 & 125.701 & 148.650 & 151.065 & 154.125 & 155.337 & 165.236 & 40,75 \\
\hline Estado & 435.594 & 434.638 & 435.735 & 456.128 & 452.564 & 444.756 & 434.604 & 429.070 & $-1,50$ \\
\hline
\end{tabular}

Fonte: SIH/SUS. TabWin: DEF=K: $\mid a i h \backslash$ tab $\backslash$ RDDRAC.DEF 
$(35,72 \%)$, Campinas $(33,44 \%)$ e Araraquara $(32,61)$. No Estado a redução foi de 12,0 para 10,8 por 1.000 hab/ano.

Quanto à participação percentual das internações por CSAP no total de internações registradas no SUS no Estado, observa-se no período uma estabilidade nos três primeiros anos, com um crescimento em 2003 (19,37\%) e, a partir daí, uma tendência permanente de redução (chegando em 2007 a 17,42\%). Observa-se uma redução em todos os DRS à exceção da Grande São Paulo, com um crescimento de $17,65 \%$ e Ribeirão Preto, com um crescimento de $0,18 \%$. Dentre os DRS em que se observou redução destacam-se os DRS de Franca (redução de 27,04\%) e de Taubaté (redução de 20,90\%).

Ao verificar a relação entre a cobertura pela Estratégia de Saúde da Família (ESF) e o percentual de internação por Condições Sensíveis à Atenção Primária (Gráfico 1), observa-se que, em 2007, a maior cobertura de ESF foi registrada no DRS de Registro (59,48\%). Entretanto, a redução das internações por CSAP nesse DRS foi pouco significativa no período, apenas $3,1 \%$. Por outro lado, a maior redução proporcional de ISAB no período foi no DRS em Franca, passando de $29,3 \%$ para $21,4 \%$ (redução de $27,0 \%$ ), cuja cobertura de ESF, em que pese ainda ser conside- rada muito baixa, registrou incremento de $141 \%$, aumentando essa cobertura de $10 \%$ para $25 \%$.

Na Tabela 3 foram desagregados os diagnósticos de forma a comparar a participação percentual de cada grupo, demonstrando sua evolução entre o ano 2000 e 2007 por DRS.

Observa-se que as internações motivadas por doenças que integram o grupo 1, que envolvem as doenças que se pode prevenir por imunização e condições sensíveis, reduziram em todos os DRS, exceto em Ribeirão Preto (aumento de 120\%) e Marília (26,61\%). As principais reduções ocorreram nos DRS de Franca $(60,26 \%)$ e Grande São Paulo (55,20\%).

As internações por gastroenterites infecciosas e complicações que compõem os diagnósticos do grupo 2, aumentaram em 4 DRS, sendo o maior aumento verificado no da Grande São Paulo (48,30\%), seguido pelo de Presidente Prudente $(23,60 \%)$.

No que diz respeito às internações por anemia (grupo 3), observa-se aumento em todos os DRS, exceto no da Baixada Santista $(9,42 \%)$ e no de Franca $(5,08 \%)$.

As internações por deficiências nutricionais, que compõem o grupo 4 , aumentaram em 4 DRS, sendo o maior aumento verificado no DRS de Presidente Prudente (46,41\%).

Tabela 2. Taxa de Internação por Condições Sensíveis à Atenção Primária Registrada no SUS, segundo DRS de Residência do Paciente. São Paulo, 2000 a 2007.

\begin{tabular}{lrrrrrrrrr}
\hline \multirow{2}{*}{ DRS } & \multicolumn{7}{c}{ Frequência de Internação Paciente Residente } & por CSAB por $\mathbf{1 . 0 0 0 h a b / a n o ~}$ & Variação \% \\
\cline { 2 - 8 } & $\mathbf{2 0 0 0}$ & $\mathbf{2 0 0 1}$ & $\mathbf{2 0 0 2}$ & $\mathbf{2 0 0 3}$ & $\mathbf{2 0 0 4}$ & $\mathbf{2 0 0 5}$ & $\mathbf{2 0 0 6}$ & $\mathbf{2 0 0 7}$ & $\mathbf{2 0 0 7 / 2 0 0 0}$ \\
\hline Grande S Paulo & 6,7 & 6,3 & 6,8 & 8,0 & 7,9 & 7,9 & 7,9 & 8,6 & 28,56 \\
Araçatuba & 17,2 & 19,2 & 19,4 & 19,3 & 17,3 & 17,4 & 16,8 & 17,2 & 0,10 \\
P. Prudente & 20,0 & 19,1 & 20,7 & 20,1 & 19,2 & 19,0 & 20,9 & 19,2 & $-4,34$ \\
Ribeirão Preto & 14,6 & 13,8 & 14,6 & 15,7 & 15,3 & 13,8 & 13,4 & 13,3 & $-9,13$ \\
Registro & 10,5 & 10,5 & 8,9 & 9,3 & 9,2 & 8,4 & 8,5 & 9,5 & $-9,92$ \\
Sorocaba & 14,4 & 14,3 & 14,1 & 14,0 & 13,5 & 12,6 & 11,9 & 11,9 & $-17,83$ \\
Bauru & 20,1 & 21,0 & 18,4 & 18,2 & 17,4 & 16,9 & 16,5 & 15,8 & $-21,64$ \\
Marília & 26,7 & 25,6 & 21,9 & 22,4 & 21,0 & 20,6 & 20,4 & 20,9 & $-21,74$ \\
S João B Vista & 22,9 & 23,5 & 22,0 & 20,7 & 19,0 & 18,3 & 17,8 & 17,9 & $-21,85$ \\
B. Santista & 11,6 & 10,4 & 10,4 & 9,7 & 10,2 & 9,0 & 9,0 & 9,1 & $-21,86$ \\
S Josén R Preto & 27,8 & 28,2 & 26,3 & 24,3 & 24,5 & 24,3 & 22,1 & 20,9 & $-24,74$ \\
Piracicaba & 11,2 & 10,8 & 10,2 & 10,5 & 10,8 & 9,2 & 8,2 & 8,0 & $-28,16$ \\
Franca & 20,7 & 20,9 & 18,4 & 18,1 & 17,0 & 15,4 & 15,2 & 14,8 & $-28,65$ \\
Barretos & 24,6 & 25,0 & 24,4 & 23,0 & 20,8 & 21,8 & 20,7 & 17,2 & $-29,97$ \\
Araraquara & 18,5 & 18,4 & 16,7 & 16,8 & 15,0 & 14,6 & 13,0 & 12,5 & $-32,61$ \\
Campinas & 12,5 & 11,0 & 11,0 & 10,6 & 10,0 & 9,4 & 8,4 & 8,3 & $-33,44$ \\
Taubatén & 14,9 & 13,9 & 13,9 & 13,3 & 11,7 & 10,8 & 9,5 & 9,6 & $-35,72$ \\
Estado & $\mathbf{1 2 , 0}$ & $\mathbf{1 1 , 6}$ & $\mathbf{1 1 , 4}$ & $\mathbf{1 1 , 8}$ & $\mathbf{1 1 , 4}$ & $\mathbf{1 1 , 0}$ & $\mathbf{1 0 , 6}$ & $\mathbf{1 0 , 8}$ & $\mathbf{- 1 0 , 1 0}$ \\
\hline
\end{tabular}

Indicador calculado com base dos dados de internação registrados no SIH/SUS e da estimativa populacional para o TCU calculada pelo IBGE (http://tabnet.datasus.gov.br/cgi/tabcgi.exe?ibge/cnv/poptuf.defo 


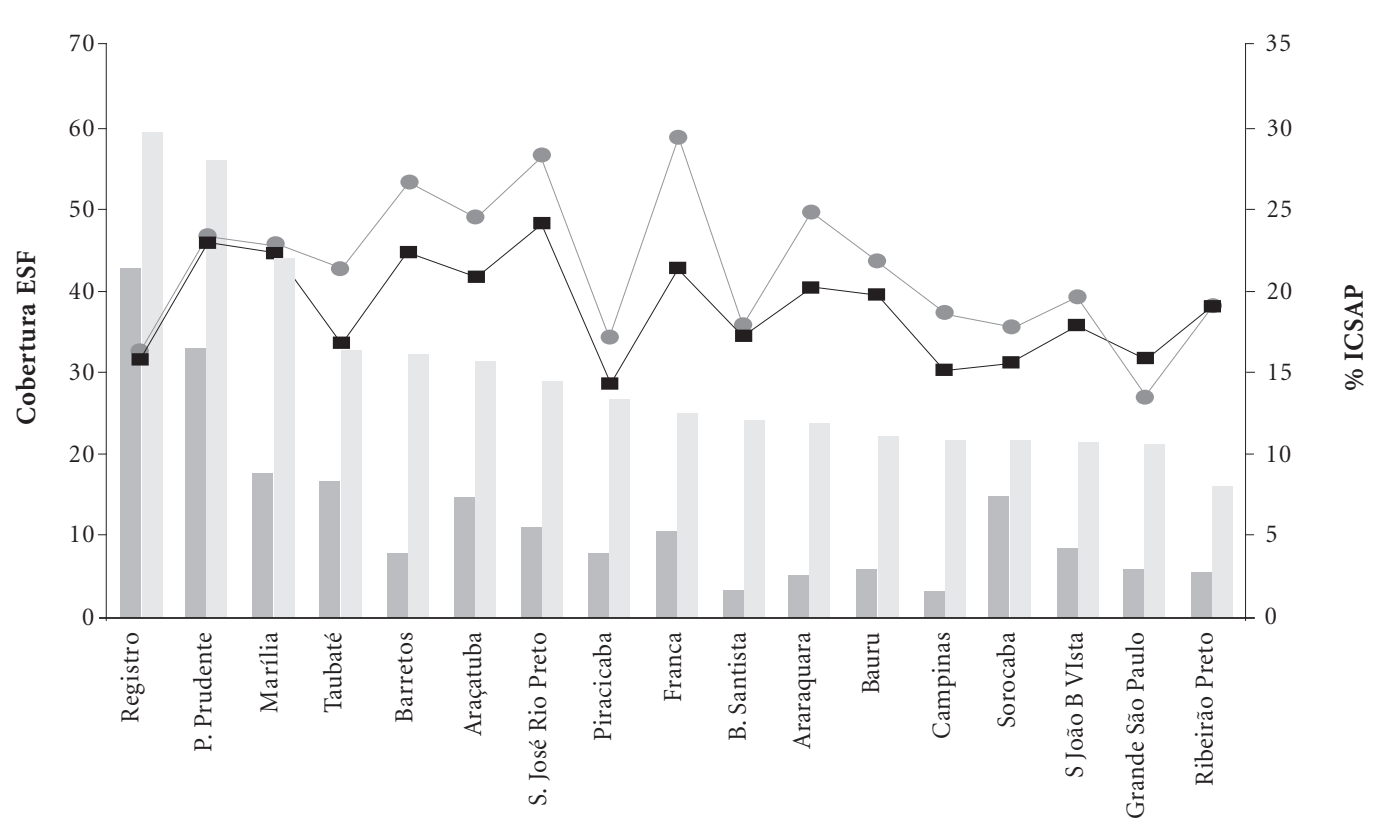

Departamentos Regionais de Saúde

Gráfico 1. Cobertura da População por Equipes de Saúde da Família e Participação das Internações por Causas Sensíveis à Atenção Primária no Total das Internações de Pacientes Residentes, segundo DRS. São Paulo, 2000 e 2007.

Fonte: SIH/SUS e SIAB/MS

As internações por infecções de ouvido, nariz e garganta (grupo 5) aumentaram apenas no DRS da Baixada Santista (15,52\%). Já as internações por pneumonias bacterianas (grupo 6) aumentaram em 11 DRS, com destaque para o da Grande São Paulo (912,24\%) e de Bauru (323,48\%).

As internações por asma (grupo 7) aumentaram em dois DRS, Ribeirão Preto $(21,04 \%)$ e Grande São Paulo (9,38\%), e as por doenças pulmonares (grupo 8) aumentaram apenas no da grande São Paulo (11,65\%).

As internações por hipertensão (grupo 9) aumentaram em 8 DRS, com destaque para o da grande São Paulo (79,94\%), e as por angina (grupo 10) aumentaram em 12 DRS, sendo a maior redução verificada nos de Registro e da Baixada Santista.

As internações por insuficiência cardíaca (grupo 11) aumentaram nos DRS da Grande São Paulo (8,47\%), Araçatuba (6,67\%) e Baixada Santista $(0,44 \%)$. Já as por doenças cerebrovasculares (grupo 12) aumentaram em 5 DRS, com destaque para o da Grande São Paulo (4,86\%).

Chamam atenção para as internações por diabetes (grupo 13) que diminuíram em todos os DRS, sendo a maior redução verificada nos de Taubaté $(43,77 \%)$, de Araçatuba $(39,97 \%)$ e de Barretos (39,85\%). As internações por epilepsias (grupo 14) aumentaram em 4 DRS, com destaque para o DRS da Grande São Paulo (158,02\%).

As internações por infecção no rim e no trato urinário (grupo 15) só diminuíram em 2 DRS: de Barretos (3,02\%) e de Marília (4,22\%).

As internações por infecção da pele e do tecido subcutâneo (grupo 16) e doença inflamatória de órgãos pélvicos femininos (grupo 17) aumentaram em 9 e 4 DRS respectivamente, com destaque para o DRS da Grande São Paulo com 74,87\% e $72,89 \%$.

As internações por úlcera gastrointestinal (grupo 18) aumentaram em 4 DRS, com destaque para o de Araçatuba, e as por doenças relacionadas ao pré- natal e parto (grupo 19) aumentaram em 8 DRS, sendo os maiores aumentos verificados no da Grande São Paulo (113,78\%) e no de Sorocaba $(110,99 \%)$.

No que diz respeito à participação dos DRS no total do valor pago de internações por CSAP, segundo dados do SIH/SUS, verifica-se que no 
Tabela 3. Variação Percentual - 2007 em Relação a 2000 - na Frequência de Internações Sensíveis à Atenção Primária, segundo Grupo de Causas e DRS de Residência no Estado de São Paulo.

\begin{tabular}{|c|c|c|c|c|c|c|}
\hline \multirow[b]{2}{*}{ Grupo de Causas (CSAP) } & \multicolumn{6}{|c|}{ Departamentos Regionais de Saúde - DRS } \\
\hline & $\begin{array}{c}\text { Grande } \\
\text { São Paulo }\end{array}$ & Araçatuba & Araraquara & $\begin{array}{l}\text { Baixada } \\
\text { Santista }\end{array}$ & Barretos & Bauru \\
\hline 1. Doenças prev. p/ imunização e cond. sensíveis & $-55,20$ & $-5,88$ & $-26,50$ & $-48,31$ & $-20,00$ & $-40,76$ \\
\hline 2. Gastroenterites infecciosas e complicações & 48,30 & $-4,98$ & $-30,49$ & $-25,99$ & $-45,14$ & $-8,77$ \\
\hline 3. Anemia & 80,79 & 98,85 & 17,76 & $-9,42$ & 7,81 & 143,37 \\
\hline 4. Deficiências nutricionais & $-35,46$ & $-18,23$ & $-28,00$ & 16,40 & $-28,57$ & $-46,79$ \\
\hline 5. Infecções de ouvido, nariz e garganta. & $-48,93$ & $-21,92$ & $-78,95$ & 15,52 & $-89,78$ & $-73,93$ \\
\hline 6. Pneumonias bacterianas & 912,24 & 147,73 & $-7,16$ & 425,20 & 170,83 & 323,48 \\
\hline 7. Asma & 9,38 & $-44,08$ & $-45,50$ & $-41,85$ & $-37,89$ & $-34,66$ \\
\hline 8. Doenças pulmonares & 11,65 & $-35,06$ & $-63,59$ & $-8,97$ & $-55,49$ & $-52,07$ \\
\hline 9. Hipertensão & 79,94 & $-6,12$ & $-42,52$ & 22,54 & $-12,31$ & $-10,33$ \\
\hline 10. Angina & 89,26 & $-2,27$ & $-14,03$ & $-34,39$ & 34,48 & 0,08 \\
\hline 11. Insuficiência cardíaca & 8,47 & 6,67 & $-21,12$ & 0,44 & $-39,73$ & $-25,16$ \\
\hline 12. Doenças cerebrovasculares & 40,86 & 10,61 & $-9,57$ & $-15,68$ & $-27,45$ & $-2,81$ \\
\hline 13. Diabetes melitus & $-7,88$ & $-9,51$ & $-39,97$ & $-30,34$ & $-39,85$ & $-34,25$ \\
\hline 14. Epilepsias & 158,02 & 6,67 & $-22,04$ & $-34,18$ & $-27,78$ & $-2,16$ \\
\hline 15. Infecção no rim e trato urinário & 110,61 & 66,80 & 49,77 & 63,25 & $-3,02$ & 87,06 \\
\hline 16. Infecção da pele e tecido subcutâneo & 74,87 & 5,95 & $-11,76$ & $-8,29$ & 38,10 & 43,80 \\
\hline 17. Doença inflamatória órgãos pélvicos femininos & 72,39 & $-43,98$ & $-45,56$ & $-25,41$ & 20,63 & $-33,49$ \\
\hline 18. Úlcera gastrointestinal & 6,05 & 34,62 & $-25,68$ & 3,20 & $-35,77$ & $-32,09$ \\
\hline 19. Doenças relacionadas ao pré-natal e parto & 113,78 & 16,67 & $-2,25$ & 77,00 & 281,48 & $-44,44$ \\
\hline Total & 40,75 & 4,63 & $-28,15$ & $-8,99$ & $-29,95$ & $-15,08$ \\
\hline
\end{tabular}

\begin{tabular}{|c|c|c|c|c|c|c|}
\hline \multirow[b]{2}{*}{ Grupo de Causas (CSAP) } & \multicolumn{6}{|c|}{ Departamentos Regionais de Saúde - DRS } \\
\hline & Campinas & Franca & Marília & Piracicaba & $\begin{array}{l}\text { Presidente } \\
\text { Prudente }\end{array}$ & Registro \\
\hline 1. Doenças prev. p/ imunização e cond. sensíveis & $-53,09$ & $-60,26$ & 26,61 & $-3,70$ & $-40,00$ & $-14,29$ \\
\hline 2. Gastroenterites infecciosas e complicações & $-54,44$ & $-49,36$ & $-19,91$ & $-48,42$ & 23,60 & 15,56 \\
\hline 3. Anemia & 20,69 & $-5,08$ & 166,67 & 14,17 & 150,72 & 21,21 \\
\hline 4. Deficiências nutricionais & $-63,22$ & 28,10 & $-28,48$ & 0,49 & 46,41 & $-42,06$ \\
\hline 5. Infecções de ouvido, nariz e garganta. & $-64,83$ & $-92,68$ & $-81,87$ & $-73,56$ & $-24,52$ & $-9,09$ \\
\hline 6. Pneumonias bacterianas & 2,00 & $-20,41$ & 18,10 & $-44,46$ & $-3,11$ & 762,50 \\
\hline 7. Asma & $-50,61$ & $-32,55$ & $-46,90$ & $-29,19$ & $-47,58$ & $-35,71$ \\
\hline 8. Doenças pulmonares & $-28,43$ & $-52,89$ & $-40,56$ & $-42,40$ & $-35,59$ & $-33,49$ \\
\hline 9. Hipertensão & 11,55 & $-32,97$ & 6,96 & 61,43 & 82,91 & $-52,78$ \\
\hline 10. Angina & 23,18 & 1,11 & 13,04 & 18,91 & 58,45 & $-46,67$ \\
\hline 11. Insuficiência cardíaca & $-26,54$ & $-27,68$ & $-18,05$ & $-46,15$ & $-2,01$ & $-14,24$ \\
\hline 12. Doenças cerebrovasculares & $-30,27$ & $-3,01$ & 35,82 & $-16,89$ & $-28,33$ & 14,89 \\
\hline 13. Diabetes melitus & $-34,04$ & $-18,37$ & $-4,19$ & $-36,86$ & $-3,14$ & $-19,02$ \\
\hline 14. Epilepsias & $-8,11$ & 11,97 & $-2,77$ & $-1,33$ & 5,79 & $-12,94$ \\
\hline 15. Infecção no rim e trato urinário & 63,59 & 29,24 & $-4,22$ & 56,76 & 52,12 & 46,12 \\
\hline 16. Infecção da pele e tecido subcutâneo & 14,23 & 45,79 & $-24,41$ & $-18,28$ & 16,82 & 23,84 \\
\hline 17. Doença inflamatória órgãos pélvicos femininos & $-48,75$ & $-26,13$ & $-64,72$ & $-30,69$ & $-8,42$ & $-3,03$ \\
\hline 18. Úlcera gastrointestinal & $-11,76$ & $-39,66$ & $-47,26$ & $-31,64$ & 2,40 & $-6,54$ \\
\hline 19. Doenças relacionadas ao pré-natal e parto & 28,72 & $-34,72$ & $-34,94$ & 53,13 & 21,15 & $-60,87$ \\
\hline Total & $-23,34$ & $-25,32$ & $-17,45$ & $-22,55$ & $-0,10$ & $-4,69$ \\
\hline
\end{tabular}




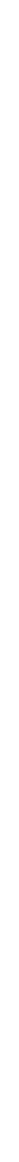

Tabela 3. continuação

\begin{tabular}{|c|c|c|c|c|c|c|}
\hline \multirow[b]{2}{*}{ Grupo de Causas (CSAP) } & \multicolumn{6}{|c|}{ Departamentos Regionais de Saúde - DRS } \\
\hline & $\begin{array}{c}\text { Ribeirão } \\
\text { Preto }\end{array}$ & $\begin{array}{l}\text { S. João da } \\
\text { Boa Vista }\end{array}$ & $\begin{array}{l}\text { S. José do } \\
\text { Rio Preto }\end{array}$ & Sorocaba & Taubaté & \\
\hline 1. Doenças prev. p/ imunização e cond. sensíveis & 120,00 & $-13,76$ & $-20,56$ & $-36,15$ & $-35,56$ & $-44,46$ \\
\hline 2. Gastroenterites infecciosas e complicações & $-11,72$ & $-16,46$ & 3,09 & $-30,23$ & $-46,27$ & $-14,64$ \\
\hline 3. Anemia & 58,18 & 306,90 & 38,92 & 167,16 & 10,82 & 64,33 \\
\hline 4. Deficiências nutricionais & $-14,50$ & $-23,52$ & $-32,41$ & $-18,89$ & $-34,21$ & $-29,30$ \\
\hline 5. Infecções de ouvido, nariz e garganta. & $-71,07$ & $-92,29$ & $-61,98$ & $-5,50$ & $-55,97$ & $-63,03$ \\
\hline 6. Pneumonias bacterianas & 60,06 & 76,44 & $-44,49$ & 84,75 & $-20,46$ & 152,24 \\
\hline 7. Asma & 21,04 & $-58,84$ & $-28,46$ & $-40,27$ & $-41,71$ & $-23,60$ \\
\hline 8. Doenças pulmonares & $-24,16$ & $-31,66$ & $-44,84$ & $-33,31$ & $-44,12$ & $-28,56$ \\
\hline 9. Hipertensão & 31,79 & 0,63 & $-12,01$ & $-30,09$ & $-39,14$ & 20,53 \\
\hline 10. Angina & 38,37 & 1,91 & 18,40 & 0,33 & $-19,68$ & 27,10 \\
\hline 11. Insuficiência cardíaca & $-41,82$ & $-35,53$ & $-30,10$ & $-5,65$ & $-11,90$ & $-13,62$ \\
\hline 12. Doenças cerebrovasculares & 18,38 & $-10,01$ & $-20,26$ & 9,11 & $-24,00$ & 6,67 \\
\hline 13. Diabetes melitus & $-34,61$ & $-20,12$ & $-36,39$ & $-32,45$ & $-43,77$ & $-23,13$ \\
\hline 14. Epilepsias & $-6,73$ & $-16,39$ & 17,24 & $-14,27$ & $-29,82$ & 27,09 \\
\hline 15. Infecção no rim e trato urinário & 58,21 & 114,90 & 3,72 & 97,31 & 34,69 & 60,37 \\
\hline 16. Infecção da pele e tecido subcutâneo & 69,80 & $-22,72$ & $-34,10$ & $-36,03$ & $-7,81$ & 29,90 \\
\hline 17. Doença inflamatória órgãos pélvicos femininos & 8,71 & $-59,46$ & $-32,86$ & 5,20 & $-35,81$ & $-9,20$ \\
\hline 18. Úlcera gastrointestinal & $-33,56$ & $-16,90$ & $-24,36$ & $-7,97$ & $-30,08$ & $-13,06$ \\
\hline 19. Doenças relacionadas ao pré-natal e parto & $-11,11$ & $-61,90$ & $-29,57$ & 110,99 & $-11,46$ & 38,45 \\
\hline Total & 0,48 & $-20,22$ & $-19,86$ & $-9,81$ & $-27,36$ & $-1,50$ \\
\hline
\end{tabular}

Fonte: SIH/SUS - TabWin: DEF=K:|aih|tab\RDDRAC.DEF

ano 2000, a maior proporção foi no da Grande São Paulo com 33,23\% do total, aumentando para 42,25\% em 2007. A menor participação foi no de Registro, com $0,44 \%$ do total em 2000, e 0,45\% em 2007. Outros DRS apresentaram no período redução na participação do total pago, destacando-se o de São João da Boa Vista, de 3,20\% para 2,3\%; o de Barretos, de 1,93\% para $1,46 \%$; e o de Taubaté, de 5,40\% para $4,22 \%$.

Ainda segundo os dados do SIH/SUS, o valor total acumulado gasto com internações por CSAP no estado de São Paulo, no período de 2000 a 2007, foi de R\$1,7 bilhões. O valor anual apresentou uma tendência de crescimento permanente no período, sendo de R \$ 167,4 milhões em 2000 e de R\$259,0 milhões em 2007.

O percentual da participação das internações por CSAP no gasto total com as internações, no estado, sofreu redução no período $(14,7 \%$ em 2000 para 13,8\% em 2007) à custa da redução verificada em 14 DRS. Ocorreu elevação na participação percentual apenas nos DRS da Grande São Paulo (10,66\%), Ribeirão Preto (7,69\%) e Registro (4,57\%).

\section{Discussão}

Ao longo dos últimos anos, a Atenção Primária (AP) foi gradualmente se fortalecendo como condição necessária para a estruturação dos sistemas locais de saúde e para a efetiva consolidação dos princípios e diretrizes do SUS.

Essa evolução se evidencia quando se compara os dados do SIAB, em que o número de municípios com Equipes de Saúde da Família, em 1994, era de apenas 55, e em setembro de 2006, quando o então Programa Saúde da Família já estava presente em 5.087 municípios. Mais significativo ainda são os dados relativos à população coberta que era de apenas 1,1 milhões de habitantes em 1994, e em 2006 já alcançava 85,5 milhões de pessoas.

Com relação aos resultados desse estudo, uma primeira questão que merece destaque é com relação à revisão da literatura onde se observa que a maioria dos trabalhos utiliza como indicador: "condições sensíveis a cuidados ambulatoriais" ao invés de utilizar "condições sensíveis à atenção primária”. 
Isso pode ter origem no fato de que os primeiros estudos relacionando internações hospitalares e atenção ambulatorial foram realizados nos Estados Unidos, onde o sistema de saúde não se caracteriza por uma organização baseada na atenção primária e nesse sentido, esses estudos tinham provavelmente como principal objetivo a redução dos custos com hospitalização.

De todo modo, apesar desse indicador ser utilizado em outros países, como por exemplo a Espanha onde o sistema de saúde tem como característica a organização da atenção primária como base, em alguns trabalhos ele também é denominado $\mathrm{ACSC}^{16}$. Por outro lado, vale ressaltar que a produção científica fora dos Estados Unidos ainda é limitada, conforme verificado na revisão de literatura realizada.

Com relação aos dados do Estado de São Paulo, embora o número de internações registradas no SUS tenha crescido $4,0 \%$ (DATASUS) no Estado, no período estudado, a cobertura de internação hospitalar sofreu uma redução, considerando que a população, no mesmo período, cresceu 9,56\% (IBGE).

Analisando os dados, observa-se que no Estado o percentual das internações por condições sensíveis à atenção primária segue uma tendência de redução no período estudado que se torna mais clara a partir dos últimos anos. Por outro lado, ao se analisar a relação entre o número de internações gerais e de internações sensíveis à atenção primária, observa-se que nos DRS onde houve crescimento de internações cresceu também o número de internações sensíveis a exemplo dos DRS de Araçatuba e Grande São Paulo. Pode-se, desta forma, supor que o aumento do acesso à internação pode contribuir para um aumento das internações sensíveis à atenção primária.

Outra questão importante é que ao analisar a relação entre as internações sensíveis à atenção primária e a cobertura da estratégia de saúde da família, pode-se inferir que existe uma relação direta, pois no período estudado houve um aumento da cobertura da ESF e redução das internações por CSAP no estado de São Paulo. Entretanto, esta relação não se deu de modo uniforme nos DRS, o que pode sustentar a hipótese de que a expansão da cobertura dessa estratégia sem a qualidade necessária não provoca um impacto sobre as internações sensíveis a atenção básica. Desse modo, pode-se supor que, embora a ampliação da cobertura da ESF no estado possa estar contribuindo para a melhoria da qualidade da $\mathrm{AB}$, talvez essa melhoria não esteja acontecendo de modo uniforme em todo território do es- tado e que também não esteja alcançando a qualidade necessária.

Entretanto, outro aspecto a ser considerado é que por se tratar de um estudo com dados agregados e ser descritivo não são estabelecidas relações de causalidade.

Ao desagregar os dados por diagnósticos, observa-se uma grande variedade de comportamento das internações segundo esses diagnósticos. Em primeiro lugar tem-se que considerar os reconhecidos problemas com a qualidade da informação no sistema de saúde brasileiro. Sabe-se que embora tenha havido uma melhoria na qualidade desses dados nos últimos anos não se pode descartar a existência de problemas, como por exemplo o registro na autorização de internação hospitalar (AIH) de diagnósticos errados ou o registro intencional de diagnóstico diferente do real com o intuito de obter maior receita do SUS.

De todo modo, observa-se que as internações por diabetes reduziram-se em todos os DRS no período. Chama a atenção que na revisão da literatura foram encontrados três trabalhos ${ }^{17-19}$ estudando isoladamente o diabetes como condição sensível. Este fato pode levantar algumas possibilidades, dentre elas de que este diagnóstico é, dentre aqueles que compõem o elenco, o mais sensível à atenção primária, ou seja, com o mínimo de organização desse nível de atenção se obtém resposta positiva. Por outro lado, as internações por hipertensão, aumentaram em oito DRS no período estudado. Este dado pode significar uma maior dificuldade para a atenção básica no controle da doença exigindo assim uma maior qualidade deste nível de atenção.

$\mathrm{Na}$ sequência, as internações que tiveram como causa os grupos de diagnósticos envolvendo as doenças que podem ser prevenidas por imunização e condições sensíveis; anemia infecção de ouvido, nariz e garganta; asma e doenças pulmonares, aumentaram em no máximo 2 DRS, o que pode significar que o mínimo de qualidade responde positivamente em função destes diagnósticos serem mais sensíveis à atenção primária.

Chama a atenção o fato das internações por doenças relacionadas ao pré-natal e ao parto (grupo 10) ter apresentado aumento em mais de 10 DRS, considerando que são diagnósticos que historicamente fazem parte da abordagem e do cuidado da atenção primária e, portanto, seria de se esperar que ocupassem outra posição neste panorama.

Considerando ainda as internações por diagnósticos que aumentaram em mais de 10 DRS, observam-se aquelas ocasionadas por pneumo- 
nias bacterianas (grupo 6) pelas infecções da pele e tecido subcutâneo (grupo 15). Este fato pode significar que a atenção primária está menos organizada para atender a estes diagnósticos ou eles são menos sensíveis a este nível de atenção?

\section{Conclusões}

Apesar das internações por condições sensíveis à atenção primária no Estado de São Paulo terem apresentado a tendência de redução ao longo do período estudado, estes resultados devem ser vistos com cautela, na perspectiva de sua adoção como indicadores da qualidade da atenção básica, pois são indicadores indiretos, com as limitações já descritas e comentadas.

Nesse sentido, um aspecto relevante a ser abordado é o fato do SIH/SUS registrar apenas as internações realizadas no âmbito do SUS e a eventual dupla ou tripla contagem de um mesmo paciente, em razão do sistema não identificar reinternações e transferências de outros hospitais.

Além disso, a impossibilidade de correções posteriores à realização do pagamento, mesmo que tenham sido identificados erros de digitação ou de codificação do diagnóstico, também se configuram em limitações a serem consideradas.

Outra questão é a publicação recente da portaria pelo Ministério da Saúde, definindo a lista brasileira de condições sensíveis à atenção pri- mária, além da não identificação de trabalhos realizados no Brasil durante a revisão da literatura, o que demonstra que ainda se desconhece o comportamento desta lista.

Apesar desses aspectos, estudos aplicando esta lista de condições sensíveis devem ser realizados, pois conforme visto na revisão bibliográfica trata-se de um indicador que mede indiretamente o funcionamento e a capacidade de resolução da Atenção Primária à Saúde.

Nesse sentido, esses estudos podem ser de grande valia para os gestores envolvidos com a atenção básica, podendo funcionar como balizadores de sua qualidade, contribuindo assim para a avaliação da implantação e da implementação das políticas de saúde no Brasil.

No campo da geração do conhecimento podem contribuir gerando hipóteses para a obtenção de respostas a partir da realização de outros estudos de avaliação de serviços, por exemplo, com desenho mais complexo e que possam agregar novos subsídios aos estudos relacionados à área.

De todo modo os resultados do presente trabalho apontam para uma melhoria no quadro de internações sensíveis à atenção primária sugerindo possível relação com a ampliação de cobertura da ESF no Estado de São Paulo. Por outro lado, vale registrar que este comportamento não se deu de modo homogêneo no estado, mas sim com grandes diferenças entre os DRS, com destaque para o da Grande São Paulo.

\section{Colaboradores}

TCMSB Rehem teve participação na concepção, análise e interpretação dos dados e redação do artigo, e EY Egry fez a revisão crítica do artigo.

\section{Agradecimentos}

Agradecemos a Tereza Cristina Lins Amaral, pelo processamento dos dados e elaboração das tabelas e gráfico. 


\section{Referências}

1. Billings J, Teicholz N. Uninsured patients in District of Columbia hospitals. Health Affairs (Millwood) 1990; 9(4):158-165.

2. Bermúdez-Tamayo C, Márquez-Calderón S, Rodríguez del Aguila MM, Perea-Milla López E, Ortiz Espinosa J. Características organizativas de la atención primaria y hospitalización por los principales ambulatory care sensitive conditions. Aten Primaria 2004; 33(6):305-311.

3. Ricketts TC, Randolph R, Howard HA, Pathman D, Carey T. Hospitalization rates as indicators of access to primary care. Health Place 2001; 7(1):27-38.

4. Gusmano MK, Rodwin VG, Weisz D. A New Way To Compare Health Systems: Avoidable Hospital Conditions In Manhattan And Paris. Health Affaairs 2006; 25(2):510-520.

5. Caminal Homar J, Starfield B, Sánchez Ruiz E, Hermosilla Pérez E, Martín Mateo M. Primary health care and hospitalizations in ambulatory care sensitive conditions in Catalonia. Rev Clin Esp 2001; 201(9):501-507.

6. Caminal Homar J, Morales Espinoza M, Sánchez Ruiz E, Cubells Larrosa MJ, Bustins Poblet M. Hospitalizaciones prevenibles mediante una atención primaria oportuna y efectiva. Aten Primaria 2003; 31(1):6-14.

7. Caminal Homar J, Casanova Matutano C. La evaluación de la atención primaria y las hospitalizaciones por ambulatory care sensitive conditions. Marco conceptual. Aten Primaria 2003; 31(1):61-65.

8. Macinko J. III Seminário Internacional de Atenção Primária - Saúde da Família. Relatório de atividades. Brasília: Ministério da Saúde (MS); 2008. [acessado 2007 dez 29]. Disponível em: http://189.28. $128.100 / \mathrm{dab} / \mathrm{docs} / \mathrm{publicacoes} /$ geral/3seminario _internacional_relatorio_atividades.pdf

9. Caminal J, Starfield B, Sánchez E, Casanova C, Morales $\mathrm{M}$. The role of primary care in preventing ambulatory care sensitive conditions. Eur J Public Health 2004; 14(3):246-251.

10. Starfield B. III Seminário Internacional de Atenção Primária - Saúde da Família. Relatório de atividades. Brasília: Ministério da Saúde (MS); 2008. [acessado 2007 dez 29]. Disponível em: http://189.28.128. $100 / \mathrm{dab} / \mathrm{docs} / \mathrm{publicacoes} /$ geral/3seminario_ internacional_relatorio_atividades.pdf
11. Brasil. Ministério da Saúde (MS). Entrevista Inês Dourado e Veneza Berenice. Revista Brasileira de Saúde da Família 2008; 18(Abr./Jun):4-6.

12. Brasil. Ministério da Saúde (MS). Consulta Pública N. 04, de setembro de 2007. Lista Brasileira de Internações Hospitalares por Condições Sensíveis à Atenção Básica. Diário oficial da União 2007; 21 set.

13. Brasil. Ministério da Saúde (MS). Publica em forma do anexo a Lista Brasileira de Internações por Condições Sensíveis à Atenção Primária. Portaria n. 221, 17 abril 2008. Diário Oficial da União 2007; 21 set.

14. Strafield B. Atenção Primária: equilíbrio entre necessidades de saúde, serviços e tecnologia. Brasília: UNESCO Brasil, Ministério da Saúde (MS); 2004.

15. Medronho RA, Carvalho DM, Bloch KV, Roner LB, Werneck V, Guilherme L. Epidemiologia. São Paulo: Editora Atheneu; 2006.

16. Valenzuela López MI, Gastón Morata JL, Melguizo Jiménez M, Valenzuela López MM, Bueno Cavanillas A. Intervenciones sanitarias en atención primaria que disminuyen la hospitalización por Ambulatory Care Sensitive Conditions en mayores de 65 años. Aten Primaria 2007; 39(10):525-532.

17. Robbins JM, Valdmanis VG, Webb DA. Do public health clinics reduce rehospitalizations?: the urban diabetes study. J Health Care Poor Underserved 2008; 19(2):562-573.

18. Niefeld MR, Braunstein JB, Wu AW, Saudek CD, Weller WE, Anderson GF. Preventable hospitalization among elderly Medicare beneficiaries with type 2 diabetes. Diabetes Care 2003; 26(5):1344-1349.

19. Booth GL, Hux JE. Relationship between avoidable hospitalizations for diabetes mellitus and income level. Arch Intern Med 2003; 163(1):101-106.

Artigo apresentado em 22/09/2009

Aprovado em 27/01/2010

Versão final apresentada em 10/02/2010 